\title{
XXV. On the reflexion of light at a twin plane of a crystal
}

\section{Lord Rayleigh Sec. R.S.}

To cite this article: Lord Rayleigh Sec. R.S. (1888) XXV. On the reflexion of light at a twin plane of a crystal , Philosophical Magazine Series 5, 26:160, 241-255, DOI: $10.1080 / 14786448808628258$

To link to this article: http://dx.doi.org/10.1080/14786448808628258

曲 Published online: 29 Apr 2009.

Submit your article to this journal $\sqsubset \pi$

山 Article views: 13

Q View related articles $₫$ 
LONDON, EDINBURGH, AND DUBLIN

\section{PHILOSOPHICAL MAGAZINE}

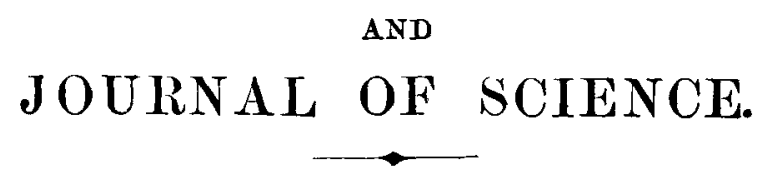

[FIFTH SERIES.]

$S E P T E M B E R \quad 1888$.

XXV. On the Reflexion of Light at a Troin Plane of a Crystal. By Lord Rayleigh, Sec. R.S.*

$\mathrm{M}^{\mathrm{Y}}$

Y object in the present paper is to calculate $a$ priori the reflexion of light at the surface between twin crystals, and to obtain formulæ analogous to those discovered by Fresnel for the case where both media are isotropic. It is evident that success can only be attained upon the basis of a theory capable of explaining at once Fresnel's laws of double refraction in crystals and those just referred to, governing the intensity of reflexion when light passes from one isotropic medium to another. So far as I am aware the electric theory of Maxwell is the only one satisfying these conditions $\dagger$; and I have accordingly employed the equations of this theory. It will be remembered that the electric theory of double refraction was worked out by Maxwell himself, and that the application to the problem of reflexion was successfully effected by von Helmholtz and Lorentz $\ddagger$. The present investigation starts, however, independently from the fundamental equations, as given in Maxwell's 'Electricity and Magnetism.'

* Communicated by the Author.

$\dagger$ See Prof. Willard Gibbs's excellent "Comparison of the Elastic and the Electrical Theories of Light with respect to the Law of Double Refraction and the Dispersion of Colours" (Am. Journ. Sci. June 1888), which reaches me while revising the present investigation for the press.

$\ddagger$ References to the works of previous writers will be found in Glazebrook's Report on Optical Theories, Brit. Assoc. Rep. 1886.

Phil. Mag. S. 5. Vol. 26. No. 160. Sept. 1888. 
Equations of a Dielectric Medium, of which the Magnetic Permeability is Unity throughout.

In Maxwell's notation the various components are represented as follows :-

Electric Displacement . . . . . . $f, g, h$;

Current . . . . . . . . . . . $u, v, w$;

Magnetic Force (or Induction) . . . . $a, b, c$;

Electromagnetic Momentum . . . . F, G, H;

Electromotive Force . . . . . . P, Q, R ;

and the equations connecting them may be written

$$
\begin{array}{r}
u=\frac{d f}{d t}, \quad v=\frac{d g}{d t}, \quad w=\frac{d h}{d t}, \quad . \quad . \quad . \\
\frac{d f}{d x}+\frac{d g}{d y}+\frac{d h}{d z}=0, . \quad . \quad . \quad . \quad . \\
4 \pi u=\frac{d c}{d y}-\frac{d b}{d z}, \quad 4 \pi v=\frac{d a}{d z}-\frac{d c}{d x}, \quad 4 \pi w=\frac{d b}{d x}-\frac{d a}{d y}, . \\
a=\frac{d \mathrm{H}}{d y}-\frac{d b}{d z}, \quad b=\frac{d \mathrm{~F}}{d z}-\frac{d \mathrm{H}}{d x}, \quad c=\frac{d b}{d x}-\frac{d \mathrm{~F}}{d y}, \quad . \\
\mathrm{P}=-\frac{d \mathrm{~F}}{d t}-\frac{d \Phi}{d x}, \quad \mathrm{Q}=-\frac{d \mathrm{G}}{d t}-\frac{d \Phi}{d y}, \quad \mathrm{R}=-\frac{d \mathrm{H}}{d t}-\frac{d \Phi}{d z} .
\end{array}
$$

In (1) it is assumed that the medium is a perfect insulator. Equations (4) and (5) may be replaced by

$$
\frac{d a}{d t}=\frac{d \mathrm{Q}}{d z}-\frac{d \mathrm{R}}{d y}, \quad \frac{d b}{d t}=\frac{d \mathrm{R}}{d x}-\frac{d \mathrm{P}}{d z}, \frac{d c}{d t}=\frac{d \mathrm{P}}{d y}-\frac{d \mathrm{Q}}{d x}, .
$$

from which $\Phi$ disappears. Thus

$$
\begin{aligned}
4 \pi \frac{d^{2} f}{d t^{2}} & =4 \pi \frac{d u}{d t}=\frac{d}{d y} \frac{d c}{d t}-\frac{d}{d z} \frac{d b}{d t} \\
& =\frac{d^{2} \mathrm{P}}{d y^{2}}+\frac{d^{2} \mathrm{P}}{d z^{2}}-\frac{d}{d x}\left\{\frac{d \mathrm{Q}}{d y}+\frac{d \mathrm{R}}{d z}\right\} \\
& =\nabla^{2} \mathrm{P}-\frac{d}{d x}\left\{\frac{d \mathrm{P}}{d x}+\frac{d \mathrm{Q}}{d y}+\frac{d \mathrm{R}}{d z}\right\} . . .
\end{aligned}
$$

where as usual

$$
\nabla^{2}=d^{2} / d x^{2}+d^{2} / d y^{2}+d^{2} / d z^{2}
$$

In ( 7$)$ and the similar equations in $g$ and $h$ there is involved no assumption as to the homogeneity or isotropy of the 
dielectric medium. If, however, these conditions are fulfilled,

$$
\frac{d \mathrm{P}}{d x}+\frac{d \mathrm{Q}}{d y}+\frac{d \mathrm{R}}{d z}=0
$$

$\mathrm{P}, \mathrm{Q}, \mathrm{R}$ being proportional to $f, g, h$; and the equations then assume a specially simple form.

The boundary conditions which must be satisfied at the transition from one homogeneous medium to another are obtained without difficulty from the differential equations. We will suppose that the surface of transition is the plane $x=0$. The first condition follows immediately from (2). It is that $f$ must be continuous across the surface $x=0$. Equation (7) shows that $d \mathrm{Q} / d y+d \mathrm{R} / d z$ must be continuous. From the similar equation in $g$, viz. :-

$$
\begin{aligned}
4 \pi \frac{d^{2} g}{d t^{2}} & =\frac{d}{d z} \frac{d a}{d t}-\frac{d}{d x} \frac{d c}{d t} \\
& =\nabla^{2} \mathrm{Q}-\frac{d}{d y}\left\{\frac{d \mathrm{P}}{d x}+\frac{d \mathrm{Q}}{d y}+\frac{d \mathrm{R}}{d z}\right\}, . . .
\end{aligned}
$$

we see not only that $d c / d t$, or $c$, must be continuous, but also that $\mathrm{Q}$ must be continuous. In like manner from the corresponding equation in $h$ it follow's that $R$ and $b$ must be continuous. T'be continuity of $\mathrm{Q}$ and $\mathrm{R}$ secures that of $d \mathrm{Q} / d y+$ $d \mathrm{R}_{j} d z$; so that it is sufficient to provide for the continuity of

$$
f, \mathrm{Q}, \mathrm{R}, \mathrm{b}, \mathrm{c} . \cdot \cdot \cdot \cdot \mathrm{A}(\mathrm{A})^{*}
$$

\section{Isotropic Reflexion.}

If both media are isotropic the problem of reflexion of plane waves is readily solved. When the electric displacements are perpendicular to the plane of incidence $(x y), f$ and $g$ vanish, while $h$ and the other remaining functions are independent of $z$. The only boundary conditions requiring attention are that $\mathrm{R}$ and $b$ should be continuous, or by $(6)$ that $\mathrm{R}$ and $d \mathrm{R} / d x$ should be continuons. This leads, as is well known, to Fresnel's sine-formula as the expression for the reflected wave.

When the electric displacements are in the plane of incidence, $h=0$, and (as before) all the remaining functions are independent of $z$. As an introduction to the more difficalt investigation before us, it may be well to give a sketch of the solution for this case. In the upper medium we have as the

* Of these conditions the first is really superfluous. If we differentiate (7) \&c. with respect to $x, y, z$ respectively and add, we see that the truth of (2) is involved. In some cases it would shorten the analytical expressions if we took $P, Q, R$ as fundamental variables, in place of $f, g, h$.

R 2 
relation between force and displacement,

$$
\mathrm{P}, \mathrm{Q}, \mathrm{R}=4 \pi \mathrm{V}^{2}(f, g, h), \text {. . . . }
$$

and in the lower,

$$
\mathrm{P}, \mathrm{Q}, \mathrm{R}=4 \pi \mathrm{V}_{1}^{2}(f, g, h), . . .
$$

$\mathrm{V}, \mathrm{V}_{1}$ being the two wave-velocities, whose ratio gives the refractive index. Since $h=0, R=0$; and since $\mathrm{R}=0$, $d \mathrm{P}^{\prime} d z=0$, it follows by (6) that $b=0$. The only conditions (A) requiring further consideration are thus the continuity of $f, \mathrm{Q}$ or $\mathrm{V}^{2} g$, and $e$.

As the expression for the incident wave we take

$$
f=q e^{i(p x+q y+s t)}, g=-p e^{i(p x+q y+s t)}, \quad . .
$$

the ratio of the coefficients being determined by the consideration that the directions $f, q, h$ and $p, q, r$ are perpendicular*. In like manner for the reflected wave we have

$$
f=q \theta^{\prime} e^{i(-p x+q y+s t)}, \quad g=p \theta^{\prime} e^{i(-p x+q y+8 t)}, \quad \text {. . }
$$

and for the refracted wave

$$
f=q \theta_{1} e^{i\left(p_{1} x+q y+s t\right)}, \quad g=-p_{1} \theta_{1} e^{i\left(p_{1} x+q y+s t\right)} .
$$

The coefficient of $y$ is the same for all the waves, since their traces on the plane $x=0$ must move together. The multipliers $\theta^{\prime}, \theta_{1}$ determine the amplitudes of the reflected and refracted waves, and may be regarded as the quantities whose expression is sought. The velocity of propagation in the first medium is $s / \sqrt{ }\left(p^{2}+q^{2}\right)$, so that

$$
\mathrm{V}^{2}\left(p^{2}+q^{2}\right)=\mathrm{V}_{1}^{2}\left(p_{1}^{2}+q^{2}\right) \ldots . . .
$$

We have now to consider the boundary conditions. The continuity of $f$, when $x=0$, requires that

$$
1+\theta^{\prime}=\theta_{1} ; \text {. . . . . . . }
$$

and the continuity of $\mathrm{V}^{2} g$ requires that

$$
\mathrm{V}^{2} p\left(1-\theta^{\prime}\right)=\mathrm{V}_{1}^{2} p_{1} \theta_{1}
$$

These two equations suffice for the determination of $\theta^{\prime}, \theta_{1}$; and we may infer that the third boundary condition is superfluous. It is easily proved to be so; for in the upper medium,

$$
\begin{aligned}
\frac{d c}{d t} & =\frac{d \mathrm{P}}{d y}-\frac{d \mathrm{Q}}{d x}=\mathrm{V}^{2}\left\{\frac{d f}{d y}-\frac{d g}{d x}\right\} \\
& =\mathrm{V}^{2}\left(1+\theta^{\prime}\right)\left(p^{2}+q^{2}\right) e^{i(q y+s t)}
\end{aligned}
$$

* In the present case $r=0$. 
when $x=0$. In the lower medium, when $x=0$,

$$
\frac{d c}{d t}=V_{1}^{2} \theta_{1}\left(p_{1}^{2}+q^{2}\right) e^{(q y+s t)} ;
$$

so that by (14) the continuity of $d c / d t$ leads to the same condition as the continuity of $f$.

The usual formula for the reflected wave is readily obtained from (15), (16). If $\phi, \phi_{1}$ be the angles of incidence and refraction,

so that

$$
\begin{aligned}
& \mathrm{V}_{1}^{2} / \mathrm{V}^{2}=\sin ^{2} \phi_{1} / \sin ^{2} \phi, \\
& p_{1} / p=\left(p_{1} / q\right) \div(p / q)=\cot \phi_{1} / \cot \phi ;
\end{aligned}
$$

Accordingly,

$$
\frac{1-\theta^{\prime}}{1+\theta^{\prime}}=\frac{\sin ^{2} \phi_{1} \cot \phi_{1}}{\sin ^{2} \phi \cot \phi}=\frac{\sin 2 \phi_{1}}{\sin 2 \phi} \text {. }
$$

$$
\theta^{\prime}=\frac{\sin 2 \phi-\sin 2 \phi_{1}}{\sin 2 \phi+\sin 2 \phi_{1}}=\frac{\tan \left(\phi-\phi_{1}\right)}{\tan \left(\phi+\phi_{1}\right)} . \quad . \quad .
$$

The insertion of this value of $\theta^{\prime}$ in (12) gives the expression for the reflected wave corresponding to the incident wave (11). The ratio of amplitudes in the two cases, being proportional to $\sqrt{ }\left(f^{2}+g^{2}\right)$, is represented by $\theta^{\prime}$, and (17) is the well-known tangent-formula of Fresnel.

\section{Propagation in a Crystal.}

In a homogeneous crystalline medium, the relation of force to strain may be expressed

$$
\mathrm{P}, \mathrm{Q}, \mathrm{R}=4 \pi\left(a_{1}^{2} f, b_{1}^{2} g, c_{1}^{2} h\right)
$$

where $a_{1}, b_{1}, e_{1}$ are the principal wave-velocities. We here suppose that the axes of co-ordinates are chosen so as to be parallel to the principal axes of the crystal. The introduction of these relations into (7), \&c., gives

where

$$
\left.\begin{array}{l}
d^{2} f / d t^{2}=a_{1}^{2} \nabla^{2} f-d \Pi / d x \\
d^{2} g / d t^{2}=l_{1}^{2} \nabla^{2} g-d \Pi / d y \\
d^{2} l / d t^{2}=c_{1}^{2} \nabla^{2} h-d \Pi / d z
\end{array}\right\}, . . .
$$

$$
\Pi=a_{1}^{2} d f / d x+b_{1}^{2} d g / d y+c_{1}^{2} d h / d z \quad . \quad .
$$

The principal problem of double refraction is the investigation of the form of the wave-surface. By means of (19) we can readily determine the law of velocity (V) for various directions of wave-front $(l, m, n)$. For this purpose we assume

$$
f, g, h=(\lambda, \mu, \nu) e^{i k \omega}, \text {. . . . . }
$$


where

$$
\omega=l x+m y+n z-\mathrm{V} t, \quad . \quad . \quad . \quad .
$$

and $k=2 \pi \div$ wave length. In accordance with (2) we must have

$$
l \lambda+m \mu+n \nu=0 \text {, . . . . . . . }
$$

signifying that the electric displacement is in the plane of the wave-front. If we now write

$$
\Pi=\Pi_{0} e^{i k \omega}
$$

and substitute the values of $f, g, h$ from (21) in (19) we find

so that by (23)

$$
\lambda\left(\mathrm{V}^{2}-a_{1}^{2}\right)=i k^{-1} \Pi_{0} . l, \& \mathrm{c} .
$$

$$
\frac{l^{2}}{\mathrm{~V}^{2}-a_{1}^{2}}+\frac{m^{2}}{\mathrm{~V}^{2}-b_{\mathrm{I}}^{2}}+\frac{n^{2}}{\mathrm{~V}^{2}-c_{1}^{2}}=0, \quad . \quad . \quad \text {. }
$$

which is Fresnel's law of velocities, leading to the wave-surface discovered by him.

\section{Reflemon at a Twin Plane.}

We are now prepared for the consideration of our special problem, viz., the reflexion of plane waves at a twin surface of a crystal. We suppose that the plane of separation is $x=0$, and we assume that there is a plane perpendicular to this $(z=0)$, with respect to which each twin is symmetrical. The only difference between the two media is that which corresponds to a rotation through $180^{\circ}$ about the axis of $x$, perpendicular to the twin plane.

In consequence of the symmetry the axis of $z$ is a principal axis in both media ; but the axes of $x$ and $y$ are not principal axes. For the relation between force and strain in the first medium we may take

$$
\mathrm{P}=4 \pi(\mathrm{A} f+\mathrm{B} g), \quad \mathrm{Q}=4 \pi(\mathrm{B} f+\mathrm{C} g), \quad \mathrm{R}=4 \pi \mathrm{D} h .
$$

In the second medium we may in the first instance assume similar expressions with accented letters; but the peculiar relation between the two media demands that $\mathrm{A}^{\prime}=\mathrm{A}, \mathrm{C}^{\prime}=\mathrm{C}$, $\mathrm{D}^{\prime}=\mathrm{D}, \mathrm{B}^{\prime}=-\mathrm{B}$. Thus for the second twin medium,

$$
\mathrm{P}=4 \pi(\mathrm{A} f-\mathrm{B} g), \mathrm{Q}=4 \pi(-\mathrm{B} f+\mathrm{C} g), \mathrm{R}=4 \pi \mathrm{D} h, .
$$

the only difference being the change in the sign of $B$. If $B$ vanish, all optical distinction between the twins disappears, and there can be no reflexion. The magnitude of $B$ depends upon the intensity of the double refraction in the twins, and also upon the angles between the principal axes and the twin 
plane. If one of these angles were to vanish, B would disappear, in spite of a powerful double refraction.

For a general solution of the problem of reflexion from a twin plane, we should have to suppose the plane of incidence to be inclined at an arbitrary angle to the plane of symmetry $(x, y)$; but we may limit ourselves without much loss of interest to the two principal cases, when the plane of incidence (1) coincides with the plane of symmetry, (2) is perpendicular to it.

\section{Incidence in the Plane of Symmetry.}

Under the first head there are two problems which may be considered separately. The simplest is that which arises when the vibrations are perpendicular to the plane of incidence, that is, are parallel to $z$. It is not difficult to see that in this case the difference between the twins never comes into operation, and that accordingly the reflexion vanishes; but it may be well to apply the general method.

Since $f, g$, and therefore [by $(25),(26)] \mathrm{P}$ and $\mathrm{Q}$, vanish throughout, while $h$ and $\mathrm{R}$ are independent of $z$, the two first of equations (7) are satisfied identically, and the third becomes

or by (25)

$$
4 \pi \frac{d^{2} h}{d t^{2}}=\frac{d^{2} \mathrm{R}}{d x^{2}}+\frac{d^{2} \mathrm{R}}{d y^{2}}
$$

$$
\frac{d^{2} h}{d t^{2}}=\mathrm{D}\left(\frac{d^{2} h}{d x^{2}}+\frac{d^{2} h}{d y^{2}}\right) . \quad \text {. . . . }
$$

This equation applies to both media, since there is no change in the value of $\mathrm{D}$. Thus, so far as the equations to be satisfied in the interior are concerned, the incident wave may be supposed to continue its course without alteration.

It is equally evident that the general boundary conditions are also satisfied. For $f, \mathrm{Q}, c$ vanish throughout, and by (6) the continuity of $R$ and $b$ merely requires the continuity of $h$ and $d h / d x$. Since all the conditions are satisfied by supposing the incident wave to pass on without alteration, it is clear that there can be no reflected waye.

We bave next to consider the case when the vibrations are executed in the plane of incidence, so that $h$ vanishes, while (as before) all the remaining functions are independent of $z$. On account of the symmetry there can be but one reflected and but one refracted wave, and in each $h$ must vanish. We may, therefore, take the following expressions as applicable to the various waves:- 
Incident wave :

satisfying

$$
f=q e^{i(p x+q y+s t)}, g=-p e^{i(p x+q y+t t)} \quad \text {. . }
$$

Reflected wave :

$$
p f+q g=0 \text {; }
$$

$$
f=q \theta^{\prime} e^{i\left(p^{\prime} x+q y+s t\right)}, \quad g=-p^{\prime} \theta^{\prime} e^{i\left(p^{\prime} x+q y+s t\right)} . .
$$

Refracted wave :

$$
f=q \theta_{1} e^{i\left(p_{1} x+q y+s t\right)}, g=-p_{1} \theta_{1} e^{i\left(p_{1} x+q y+s t\right)} . .
$$

The coefficient of the time $(s)$ is necessarily the same throughout on account of the periodicity ; and the coefficient of $y$ is the same, since the traces of all three waves upon the plane of separation $x=0$ must move together. The relations between $p, q, s ; p^{\prime}, q, s ; p_{1}, q, s$ are to be obtained by substitution in the differential equations. Of these the equation in $h$ is satisfied identically, since $\mathrm{R}=0$. The other equations for the upper medium are by (7), (8), (25),

$$
\begin{aligned}
& \frac{d^{2} f}{d t^{2}}=\frac{d^{2}}{d y^{2}}(\mathrm{~A} f+\mathrm{B} g)-\frac{d^{2}}{d x d y}(\mathrm{~B} f+\mathrm{C} g), \\
& \frac{d^{2} g}{d t^{2}}=\frac{d^{2}}{d x^{2}}(\mathrm{~B} f+\mathrm{C} g)-\frac{d^{2}}{d x d y}(\mathrm{~A} f+\mathrm{B} g) .
\end{aligned}
$$

These must be satisfied by the incident and reflected waves. On substitution we find that both equations lead to the same conditions, viz. :-

$$
s^{2}=\mathrm{A} q^{2}-2 \mathrm{~B} p q+\mathrm{C} p^{2}, \quad . \quad . \quad .
$$

a quadratic equation of which the two roots give $p$ and $p^{\prime}$ in terms of $q$ and $s$.

In the second medium we get in like manner for the refracted wave

$$
s^{2}=\mathrm{A} q^{2}+2 \mathrm{~B} p_{1} q+\mathrm{C}_{1}{ }^{2}, \text {. . . . }
$$

the sign of $\mathrm{B}$ being changed. Equating the two values of $s^{2}$, we find

or

$$
\begin{aligned}
& \mathrm{C}\left(p^{2}-p_{1}^{2}\right)=2 \mathrm{~B} q\left(p+p_{1}\right), \\
& \mathrm{C}\left(p-p_{1}\right)=2 \mathrm{~B} q . . . . . .
\end{aligned}
$$

We have now to consider the boundary conditions (A). The functions $R$ and $b$ vanish throughout; but it remains to provide for the continuity of $f, \mathrm{Q}$, and $c$, when $x=0$. The first of these conditions gives at once

$$
1+\theta^{\prime}=\theta_{1} \text {. . . . . . . }
$$

Again, the continuity of $\mathrm{Q}$, equal to $\mathrm{B} f+\mathrm{C} g$ in the first 
medium, and to $-\mathrm{B} f+\mathrm{C} g$ in the second, gives

$$
\mathrm{B} q-\mathrm{C} p+\theta^{\prime}\left(\mathrm{B} q-\mathrm{C} p^{\prime}\right)=-\theta_{1}\left(\mathrm{~B} q+\mathrm{C} p_{1}\right) . .
$$

The continuity of $c$ leads, when regard is paid to (31), (32), merely to the repetition of the condition (34).

If we eliminate $\theta_{1}$ between (34), (35), we find

$$
\theta^{\prime}\left\{2 \mathrm{~B} q-\mathrm{C} p^{\prime}-\mathrm{C} p_{1}\right\}=\mathrm{C}\left(p-p_{1}\right)-2 \mathrm{~B} q=0 \text { by (33). }
$$

Hence $\theta^{\prime}$ vanishes. Neither in this case, nor when the vibrations are perpendicular to the plane of incidence, is there any reflexion of light incident in the plane of symmetry. And this conclusion may of course be extended to natural light, and to light plane or elliptically polarized in any way whatever.

\section{Plane of Incidence perpendicular to that of Symmetry.}

We have now to consider the case when the plane of incidence is the plane $y=0$, perpendicular to that of symmetry. Here $f, g, l$ are all finite, but they (as well as $\mathrm{P}, \mathrm{Q}, \mathrm{R}, \mathrm{\& c}$.) are independent of the coordinate $y$. The problem is more complicated than when the plane of incidence coincides with that of symmetry, because an incident wave is here attended by two reflected waves, and two refracted waves.

The equation of the incident wave in the upper medium may be expressed

$$
\begin{aligned}
f, g, h & =(\lambda, \mu, \nu) e^{i(p x+r z+s t)} ; \\
\text { or, since by (2) } \lambda p+\nu r & =0, \\
f, g, h & =(r, \mu,-p) e^{i(p x+r z+s t)} .
\end{aligned}
$$

The differential equations to be satisfied in the upper medium assume the form

$$
\begin{aligned}
& \frac{d^{2} f}{d t^{2}}=\frac{d^{2}}{d z^{2}}(\mathrm{~A} f+\mathrm{B} g)-\frac{d^{2}}{d x d z}(\mathrm{D} h), \\
& \frac{d^{2} g}{d t^{2}}=\left(\frac{d^{2}}{d x^{2}}+\frac{d^{2}}{d z^{2}}\right)(\mathrm{B} f+\mathrm{C} g), \\
& \frac{d^{2} h}{d t^{2}}=\frac{d^{2}}{d x^{2}}(\mathrm{D} h)-\frac{d^{2}}{d x d z}(\mathrm{~A} f+\mathrm{B} g) .
\end{aligned}
$$

If we substitute for $f, g, h$ from (36), the first and third equations give

$$
s^{2}=r(\mathrm{~A} r+\mathrm{B} \mu)+p^{2} \mathrm{D}, \quad . \quad . \quad .
$$

and the second equation gives

$$
\mu s^{2}=\left(p^{2}+r^{2}\right)(\mathrm{B} r+\mathrm{C} \mu) . . .
$$


These two equations determine $p$ and $\mu$, when $r, s$ are given. Since the elimination of $\mu$ leads to a quadratic in $p^{2}$, it is evident that there are four admissible values $\pm p_{1}, \pm p_{2}$, corresponding to waves of given periodicity, whose trace on the plane of separation moves with a given velocity. Of these two (say with the + sign) are waves approaching the surface, and two are waves receding from it. If we limit ourselves to a single incident wave $\left(+p_{1}\right)$, we shall have still to take into account two reflected waves corresponding to $-p_{1},-p_{2}$. The equations show that the value of $\mu$ is the same whether $p$ be positive or negative; we shall suppose that $\mu_{1}$ corresponds to $\pm p_{1}, \mu_{2}$ to $\pm p_{2}$.

In applying the equations to the second medium we have to change the sign of $B$; and it is evident that they are satisfied by the same values of $p$ as before, and that the preceding values of $\mu$ are to be taken negatively. Hence in the second medium $-\mu_{1}$ corresponds to $\pm p_{1},-\mu_{2}$ to $\pm p_{2}$. For the purposes of our present problem, where there is no incident wave in the second medium, we are concerned only with $+p_{1}$ and $+p_{2}$.

The complete specification of the system of waves corresponding to a single incident wave $\left(p_{1}\right)$ in the first medium is thus :-

Incident wave :

$$
f, g, h=\left(r, \mu_{1},-p_{1}\right) \Theta_{1} e^{i\left(p_{1} x+r z+s t\right)} ; . . .
$$

Two reflected waves:

$$
\begin{aligned}
f, g, h & =\left(r, \mu_{1}, p_{1}\right) \theta^{\prime} e^{i\left(-p_{1} x+r z+s t\right)} \\
& +\left(r, \mu_{2}, p_{2}\right) \theta^{\prime \prime} e^{i\left(-p_{2} x+r z+s t\right)} ; \quad . .
\end{aligned}
$$

Two refracted ${ }^{*}$ waves :

$$
\begin{aligned}
f, g, h & =\left(r,-\mu_{1},-p_{1}\right) \theta_{1} e^{i\left(p_{1} x+r z+8 t\right)} \\
& +\left(r,-\mu_{2},-p_{2}\right) \theta_{2} e^{i\left(p_{2} x+r z+\theta t\right)} . \quad . .
\end{aligned}
$$

The next step is the introduction of the boundary conditions (A). The continuity of $f$ requires that

$$
\Theta_{1}+\theta^{\prime}+\theta^{\prime \prime}=\theta_{1}+\theta_{2} \text {. . . . . . }
$$

The continuity of $\mathrm{R}$, or $\mathrm{D} h$, or $h$, gives with equal facility

$$
p_{1} \Theta_{1}-p_{1} \theta^{\prime}-p_{2} \theta^{\prime \prime}=p_{1} \theta_{1}+p_{2} \theta_{2} . \quad \text {. . . }
$$

Again, the continuity of $\mathrm{Q}$, equal to $\mathrm{B} f+\mathrm{C} g$ in the first

* It should be noticed that one of the refracted wares is not refracted in the literal sense, being parallel to the incident wave. 
medium and to $-\mathrm{B} f+\mathrm{C} g$ in the second, gives

$$
\begin{aligned}
\left(\mathrm{B} r+\mathrm{C} \mu_{1}\right) \Theta_{1}+\left(\mathrm{B} r+\mathrm{C} \mu_{1}\right) \theta^{\prime}+\left(\mathrm{B} r+\mathrm{C} \mu_{2}\right) \theta^{\prime \prime} & \\
& =-\left(\mathrm{B} r+\mathrm{C} \mu_{1}\right) \theta_{1}-\left(\mathrm{B} r+\mathrm{C} \mu_{2}\right) \theta_{2} . .
\end{aligned}
$$

The continuity of $b$, or $d b / d t$, or by (6) $d \mathrm{R} / d x-d \mathrm{P} / d z$, is found, when regard is paid to (37), to be already secured by (42); and we bave only further to consider the continuity of $d c / d t$, or by (b) of $d \mathrm{Q} / d x$, since $\mathrm{P}$ is here independent of $y$. Thus

$$
\begin{gathered}
p_{1}\left(\mathrm{~B} r+\mathrm{C} \mu_{1}\right) \Theta_{1}-p_{1}\left(\mathrm{~B} r+\mathrm{C} \mu_{1}\right) \theta^{\prime}-p_{2}\left(\mathrm{~B} r+\mathrm{C} \mu_{2}\right) \theta^{\prime \prime} \\
=-p_{1}\left(\mathrm{~B} r+\mathrm{C} \mu_{1}\right) \theta_{1}-p_{2}\left(\mathrm{~B} r+\mathrm{C} \mu_{2}\right) \theta_{2} .
\end{gathered} .
$$

The coefficients which occur in (44), (45) may be expressed more briefly in terms of the velocities of the various waves. For

and thus by (38),

$$
\mathrm{V}^{2}=s^{2} /\left(p^{2}+r^{2}\right), \quad \cdot \quad \cdot \quad \cdot \quad \cdot
$$

Setting now

$$
\mathrm{B} r+\mathrm{C} \mu_{1}=\mu_{1} \mathrm{~V}_{1}^{2}, \quad \mathrm{~B} r+\mathrm{C} \mu_{2}=\mu_{2} \nabla_{2}^{2} \cdot .
$$

$$
p_{2} / p_{1}=\varpi, \quad \mu_{2} \mathrm{~V}_{2}^{2} / \mu_{1} \mathrm{~V}_{1}^{2}=\sigma, \quad . \quad .
$$

the four equations of condition take the form

$$
\left.\begin{array}{l}
\Theta_{1}+\theta^{\prime}+\theta^{\prime \prime}=\theta_{1}+\theta_{2}, \\
\Theta_{1}-\theta^{\prime}-\varpi \theta^{\prime \prime}=\theta_{1}+\varpi \theta_{2}, \\
\Theta_{1}+\theta^{\prime}+\sigma \theta^{\prime \prime}=-\theta_{1}-\sigma \theta_{2}, \\
\Theta_{1}-\theta^{\prime}-\varpi \sigma \theta^{\prime \prime}=-\theta_{1}-\varpi \sigma \theta_{2} .
\end{array}\right\} . \cdot .
$$

If we equate the values of $\theta_{1}, \theta_{2}$ obtained from the first and second pairs of equations (48), we find

$$
\left.\begin{array}{r}
(\sigma+1) \theta^{\prime}+(\varpi+1) \sigma \theta^{\prime \prime}=0, \\
(\varpi-1) \Theta_{1}+(\varpi+1) \theta^{\prime}+\varpi(\sigma+1) \theta^{\prime \prime}=0,
\end{array}\right\} .
$$

and from these again

$$
\begin{aligned}
& \theta^{\prime}=-\frac{\sigma\left(\varpi^{2}-1\right) \Theta_{1}}{(\varpi-\sigma)(\varpi \sigma-1)}, \quad . \quad . \quad . \quad . \\
& \theta^{\prime \prime}=\frac{(\sigma+1)(\varpi-1) \Theta_{1}}{(\varpi-\sigma)(\varpi \sigma-1)}, \ldots . . .
\end{aligned}
$$

by which the two reflected waves are determined.

These reflected waves correspond to the incident wave $\left(\Theta_{1}, p_{1}, \mu_{1}\right)$, and it is the wave $\theta^{\prime}$ which is reflected according to the ordinary law. If there be a second incident wave $\left(\Theta_{2}, p_{2}, \mu_{2}\right)$, the corresponding reflected waves are to be found from (50), (51) by interchanging $\theta^{\prime}, \theta^{\prime \prime}$, and by writing for $\sigma, \sigma$ the reciprocals of these ratios. If both incident waves 
coexist,

$$
\begin{aligned}
& \theta^{\prime}=\frac{(1-\sigma) \sigma}{(\varpi-\sigma)(\varpi \sigma-1)}\left\{(1+\sigma) \Theta_{1}+\varpi(1+\sigma) \Theta_{2}\right\}, . \\
& \theta^{\prime \prime}=\frac{\varpi-1}{(\varpi-\sigma)(\varpi \sigma-1)}\left\{(1+\sigma) \Theta_{1}+(1+\varpi) \sigma \Theta_{2}\right\} .
\end{aligned}
$$

It will be observed that although the fronts of the two incident waves $\Theta_{1}, \Theta_{2}$ are not parallel, they are the waves that would be generated by the double refraction of a single wave incident from an isotropic medium upon a face of the crystal parallel to the twin plane.

\section{Doubly Refracting Power small.}

Thus far our equations are general. But the interpretation will be very much facilitated if we introduce a supposition, which does not deviate far from the reality of nature, viz. that the doubly refracting energy is comparatively small. There is no new limitation upon the direction of the principal axes relatively to those of coordinates, but we assume that $\mathrm{A}, \mathrm{C}, \mathrm{D}$ are nearly equal, and that $B$ is small. We may imagine the two twin crystals to be bounded by faces parallel to the twin face, and to be embedded in an isotropic medium of nearly similar optical power. Under these circumstances $p_{1}, p_{2}$; $V_{1}, V_{2}$ are nearly equal, so that approximately $\sigma=1, \sigma=\mu_{2} / \mu_{1}$; and we may write (52), (53) in the form

$$
\begin{aligned}
& \theta^{\prime}=\frac{p_{2}-p_{1}}{p\left(\mu_{2}-\mu_{1}\right)^{2}}\left\{2 \mu_{1} \mu_{2} \Theta_{1}+\mu_{2}\left(\mu_{1}+\mu_{2}\right) \Theta_{2}\right\}, \quad . . \\
& \theta^{\prime \prime}=\frac{p_{1}-p_{2}}{p\left(\mu_{2}-\mu_{1}\right)^{2}}\left\{\mu_{1}\left(\mu_{1}+\mu_{2}\right) \Theta_{1}+2 \mu_{1} \mu_{2} \Theta_{2}\right\} . .
\end{aligned}
$$

It should be remarked that the intensities of the waves represented by $\Theta_{1}$ \&c. are not simply proportional to $\Theta_{1}{ }^{2}$, \&c. Referring to (39), (40), we see that the intensity of $\Theta_{1}, \theta^{\prime}$ is measured by $\left(r^{2}+p^{2}+\mu_{1}^{2}\right)\left(\Theta_{1}^{2}, \theta^{\prime 2}\right)$; and that of $\Theta_{2}, \theta^{\prime \prime}$ by $\left(r^{2}+p^{2}+\mu_{2}^{2}\right)\left(\Theta_{2}^{2}, \theta^{\prime \prime 2}\right)$.

\section{Plate bounded by Surfaces parallel to Twin Plane.}

Let us now regard the waves $\Theta_{1}, \Theta_{2}$ as due to the passage into the crystal of waves from an isotropic medium, under such conditions (of gradual transition, if necessary) that there is no loss by reflexion. The interface is supposed to be parallel to the twin reflecting plane, and the optical power to be so nearly equal to that of the crystal that the refraction is negligible. Then, if the vibration parallel to $y$ (perpen- 
dicular to the plane of incidence) be $\mathrm{M}$, and that in plane of incidence be $\mathrm{N}$, we have

$$
\begin{aligned}
& \mathrm{M}=\mu_{1} \Theta_{1}+\mu_{2} \Theta_{2}, \quad . \quad . \quad . \quad \\
& \mathrm{N}=\sqrt{ }\left(p^{2}+r^{2}\right)\left\{\Theta_{1}+\Theta_{2}\right\} .
\end{aligned}
$$

In like manner, if the vibrations of the emergent reflected wave perpendicular and parallel to the plane of incidence be $\mathbf{M}^{\prime}, \mathbf{N}^{\prime}$

$$
\begin{aligned}
& \mathrm{M}^{\prime}=\mu_{1} \theta^{\prime}+\mu_{2} \theta^{\prime \prime}, \quad . \quad . \quad . \quad . . \\
& \mathrm{N}^{\prime}=\sqrt{ }\left(p^{2}+r^{2}\right)\left\{\theta^{\prime}+\theta^{\prime \prime}\right\} .
\end{aligned}
$$

If we are prepared to push to an extreme our supposition as to the smallness of the doubly refracting power, $\Theta, \theta$ in these equations may be identified with the corresponding quantities in (54), (55); for a retardation of phase in crossing and recrossing the stratum alike for all the waves might be disregarded. We shall presently return to this question; but we will in the meantime trace out the consequences which ensue when the double refraction, if not extremely small in itself, is at least so small in relation to the distances through which it acts (the thickness of the stratum), that the relative changes of phase may be neglected. Then

$$
\begin{aligned}
\mathrm{M}^{\prime} & =\frac{\left(p_{2}-p_{1}\right) \mu_{1} \mu_{2}}{p\left(\mu_{1}-\mu_{2}\right)}\left\{\Theta_{1}+\Theta_{2}\right\} \\
& =\frac{\left(p_{2}-p_{1}\right) \mu_{1} \mu_{2}}{p\left(\mu_{1}-\mu_{2}\right)} \frac{\mathrm{N}}{\sqrt{\left(p^{2}+r^{2}\right.}}, \quad . . . \\
\mathrm{N}^{\prime} & =\frac{\left(p_{2}-p_{1}\right) \sqrt{ }\left(p^{2}+r^{2}\right) . \mathrm{M}}{p\left(\mu_{2}-\mu_{1}\right)} . . . .
\end{aligned}
$$

We have now to introduce certain relations derived from (37), (38). By elimination of $s$, we get

$$
\mathrm{B} r . \mu^{2}+\mu\left\{(\mathrm{A}-\mathrm{C}) r^{2}+(\mathrm{D}-\mathrm{C}) p^{2}\right\}-\mathrm{B} r\left(p^{2}+r^{2}\right)=0 .
$$

If we here disregard the difference between $p_{1}$ and $p_{2}$, we may treat it as a quadratic, by which the two values of $\mu$ are determined ; and it follows that

$$
-\mu_{1} \mu_{2}=p^{2}+r^{2} \text {. }
$$

We might have arrived at this conclusion more quickly from the consideration that in the limit the two directions of displacement $\left(r, \mu_{1}, p_{1}\right),\left(r, \mu_{2}, p_{2}\right)$ in the reflected waves must be perpendicular to one another.

Again, from the general equation (37) we see that

$$
\mathrm{B} r\left(\mu_{1}-\mu_{2}\right)+\left(p_{1}{ }^{2}-p_{2}{ }^{2}\right) \mathrm{D}=0 \text {, }
$$


whence approximately,

$$
\frac{p_{2}-p_{1}}{\mu_{1}-\mu_{2}}=\frac{r \mathrm{~B}}{2 p \mathrm{D}} . . .+. .
$$

Introducing these relations inio (60), (61), we find

$$
\begin{aligned}
& \mathrm{M}^{\prime}=-\frac{r \sqrt{ }\left(p^{2}+r^{2}\right) \cdot \mathrm{B} \cdot \mathrm{N}}{2 p^{2} \mathrm{D}}, \quad . \quad . \\
& \mathrm{N}^{\prime}=-\frac{r \sqrt{ }\left(p^{2}+r^{2}\right) \cdot \mathrm{B} \cdot \mathrm{M}}{2 p^{2} \mathrm{D}} \cdot . . .
\end{aligned}
$$

These equations indicate that the intensity of the reflected light $\left(\mathrm{M}^{\prime 2}+\mathrm{N}^{\prime 2}\right)$ is proportional to that of the incident, without regard to the polarization of the latter. Again, if the incident light be unpolarized ( $M$ and $N$ equal, and without permanent phase relation), so also is the reflected light. But what is more surprising is, that if the incident light be polarized in or perpendicular to the plane of incidence, the reflected light is polarized in the opposite manner.

The intensity of reflexion may be expressed in terms of the angle of incidence $\phi$, for

so that

$$
p / \sqrt{ }\left(p^{2}+r^{2}\right)=\cos \phi, \quad r / \sqrt{ }\left(p^{2}+r^{2}\right)=\sin \phi,
$$

$$
\mathrm{M}^{12}+\mathrm{N}^{\prime 2}=\frac{\mathrm{B}^{2} \sin ^{2} \phi}{4 \mathrm{D}^{2} \cos ^{4} \phi}\left(\mathrm{M}^{2}+\mathrm{N}^{2}\right) . \quad \text {. . . }
$$

When the angle of incidence is small, the intensity is proportional to its square. And, as was to be expected, the reflexion is proportional to $B^{2}$.

The laws here arrived at are liable to modification when, as must usually bappen in practice, the thickness of the plate cannot be neglected. The incident light, on its way to the twin surface, and the reflected light on its way back, is subject to a depolarizing influence, which in most cases complicates the relation between the polarizations of the light before entering and after leaving the crystal. One law, however, remains unaffected. If the light impinging upon the crystal be unpolarized, it retains this character upon arrival at the twin face. We have shown that it does not lose it in the act of reflexion, neither can it lose it in the return passage after reflexion. Hence, if the light originally incident upon the layer of crystal be unpolarized, so is the reflected light ultimately emergent from it. 
If, on the other hand, the incident light be polarized, whether plane or elliptically, the character of the emergent light must depend upon the precise thickness of the crystalline layer, and will vary rapidly from one part of the spectrum to another. The simplest case that we can consider is when the polarization of the incident rays is such that one or other of $\Theta_{1}, \Theta_{2}$ vanish. We will suppose that it is $\Theta_{2}$; so that after reflexion,

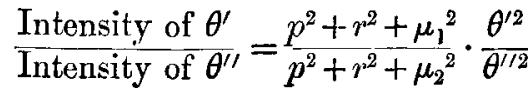

$$
\begin{aligned}
& =\frac{\mu_{1}^{2}-\mu_{1} \mu_{2}}{\mu_{2}^{2}-\mu_{1} \mu_{2}} \frac{4 \mu_{1}^{2} \mu_{2}^{2}}{\mu_{1}^{2}\left(\mu_{1}+\mu_{2}\right)^{2}}=\frac{-4 \mu_{1} \mu_{2}}{\left(\mu_{1}+\mu_{2}\right)^{2}} .
\end{aligned}
$$

by (54), (55). This is the ratio of intensities that would be observed with an analyzing nicol held so as to retain in succession $\theta^{\prime}$ and $\theta^{\prime \prime}$. If the crystalline layer be moderately thick, and the light be of mixed wave-lengths, there will be no interference observable between $\theta^{\prime}$ and $\theta^{\prime \prime}$, and thus the ratio just found is the extreme ratio of intensities. By means of (62) we may express it in terms of the angle of incidence $(\phi)$, and of the fundamental optical constants of the crystal. ihus

$$
\begin{gathered}
\sqrt{\left\{\frac{-\mu_{1} \mu_{2}}{\left(\mu_{1}+\mu_{2}\right)^{2}}\right\}=\frac{\sqrt{ }\left\{p^{2}+r^{2}\right\} \cdot \mathrm{B} r}{(\mathrm{~A}-\mathrm{C}) r^{2}+(\mathrm{D}-\mathrm{C}) p^{2}}} \\
=\frac{\mathrm{B} \sin \phi}{(\mathrm{A}-\mathrm{C}) \sin ^{2} \phi+(\mathrm{D}-\mathrm{C}) \cos ^{2} \phi} .
\end{gathered}
$$

This expression shows that in general the emergent light will be fully polarized only when $\phi$ is very small. In this case we virtually fall back upon our original investigation where the thickness of the layer was neglected. Since only $\Theta_{1}$ is present, there is no depolarization in the first passage ; and when $\phi=0$ there is no depolarization upon the return passage in consequence of the disappearance of $\theta^{\prime}$. The polarizations corresponding in this case to $\Theta_{1}, \Theta_{2}$ are obviously those in and perpendicular to the plane of incidence; and we learn that, when the angle of incidence is small, polarizations in and perpendicular to the plane of incidence are reversed in the reflected ray. If the incident ray be polarized in other directions than these, the reflected ray is in general not fully polarized, even though the angle of incidence be small. 